(C) [2008] IEEE. Reprinted, with permission, from [Howarth, Blair; Katupitiya, J; Eaton, Ray; Kodagoda, Sarath. Localization of visually indistinguishable mature lettuce heads using spatial information, Mechatronics and Machine Vision in Practice, 2008. M2VIP 2008. 15th International Conference]. This material is posted here with permission of the IEEE. Such permission of the IEEE does not in any way imply IEEE endorsement of any of the University of Technology, Sydney's products or services. Internal or personal use of this material is permitted. However, permission to reprint/republish this material for advertising or promotional purposes or for creating new collective works for resale or redistribution must be obtained from the IEEE by writing to pubs-permissions@ieee.org. By choosing to view this document, you agree to all provisions of the copyright laws protecting it 


\title{
Localization of Visually Indistinguishable Mature Lettuce Heads Using Spatial Information
}

\author{
Blair Howarth ${ }^{1}$, Jayantha Katupitiya ${ }^{1}$, Ray Eaton ${ }^{2}$, Sarath Kodagoda ${ }^{3}$ \\ ARC Centre of Excellence for Autonomous Systems, Sydney, Australia \\ ${ }^{1}$ School of Mechanical and Manufacturing Engineering, The University of New South Wales \\ ${ }^{2}$ School of Electrical Engineering and Telecommunications, The University of New South Wales \\ ${ }^{3}$ University of Technology Sydney
}

\begin{abstract}
This paper describes an approach to recognizing and localizing centers of mature lettuce heads in the field when the lettuce leaves obscure the distinctions between plants. This is of great value when using an automatic harvester in cluttered or closely planted vegetation. The aim of this work is to investigate and verify the potential use of spatial rather than visual clues for recognition and localisation, with a view to implementing a more robust and sophisticated system if promise is shown. Colour/texture information was difficult to use so spatial information was used instead. A laser range finder was used to generate a height plot from above the plants. Lettuce examples were used to learn the radial distribution of the lettuce model. This was compared with the distributions of arbitrary locations in new scans to locate possible lettuce locations. Planting distance information was then used to localize the final lettuce positions. The algorithm was able to successfully locate 15 out of 16 sample lettuces.
\end{abstract}

\section{INTRODUCTION}

Automatic harvesting of vegetation is currently an attractive area of research. The problem being addressed in this paper relates to the accurate localization of mature lettuce heads in a very cluttered and visually unrecognizable environment. Even when the locations where the lettuce have been planted are known exactly, when they grow, the mature lettuce head may deviate by as much as $70 \mathrm{~mm}$ or more in any given direction. The lettuce harvesting process requires a maturity test by pressing on the center of the lettuce head to carry out a stiffness measurement. An average lettuce is $170 \mathrm{~mm}-200 \mathrm{~mm}$ in diameter so a $70 \mathrm{~mm}$ deviation is too large to successfully carry out the maturity test before harvesting. Hence there is a need to accurately determine the centers of fully grown lettuce heads. A successful system would alleviate the need for manual localization and harvesting, which is very labour intensive.

Most automated vegetation recognition systems attempt to use vision systems which are responsible for recognizing and locating the individual plants so that they can be harvested in some fashion. In many cases this is a trivial task as the plants are well spaced and visual information provides a good contrast between the plant and background.

The most common application of vegetation recognition and localization in the literature is for weed control. The general approach taken involves gray scale visual image segmentation to contrast the background from the plants. Next morphological shape features of the 2D silhouettes are used to distinguish between vegetation and weeds. Examples of this approach can be found in [1] and [2]. A real-time algorithm was implemented to detect weeds amongst tomato plants in [3]. The accuracy of a process based on shape features was moderate with a high level of both false positive and false negative detections.

Colour information was used to provide extra visual features in [4] to locate weeds amongst sugar beet plants. The use of colour properties along with shape information proved to be very useful in discriminating crop from weeds and resulted in a successful classifier with a high detection rate. Recognition of lettuce has been attempted by [5]. They also used shape information to locate weeds amongst lettuce, however the planting separation was significant and so the plants could easily be segmented visually. Colour information was used by [6] to localize radicchio lettuce plants. They used colour images to take advantage of the contrast between the maroon coloured head of this particular species of lettuce and the green leaves. They were able to successfully localize lettuce even if the head was occluded

This visual segmentation approach is viable in these situations because the weed control is started soon after planting. The plants and weeds occupy only a small fraction of the total ground resulting in easily detectable differences between dirt and plants. Even as the plants grow to maturity this contrast generally remains.

However in situations where there is no clear distinction between neighboring plants or background, this method becomes difficult or impossible to apply. An example of this is a field of mature lettuce that are planted closely together. The leaves of neighboring plants overlap removing the contrast between plant and background. The colour and texture of the leaves are very similar to that of the lettuce head, or are still covering the head, making visual information almost useless. Figure 1 shows an example of this. The lettuces are difficult to detect visually as some leaves are still covering the lettuce head whilst other leaves are not. Figure 2 shows the locations of the lettuces for comparison.

Our approach to this problem is instead based on spatial information acquired by a laser range finder. The lettuces can be modeled spatially in $3 \mathrm{D}$ and this provides much more useful information for recognition. The main aim of this paper is the investigation and verification of the potential use of spatial information as opposed to visual clues for recognition and localisation. If promise is shown then a more 


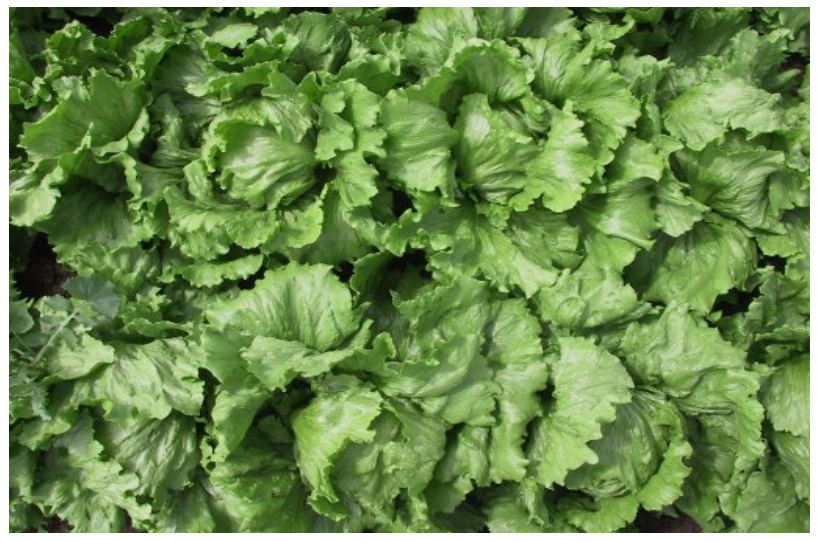

Fig. 1. Image of lettuce, illustrating the difficulty of using colour or texture information for recognition

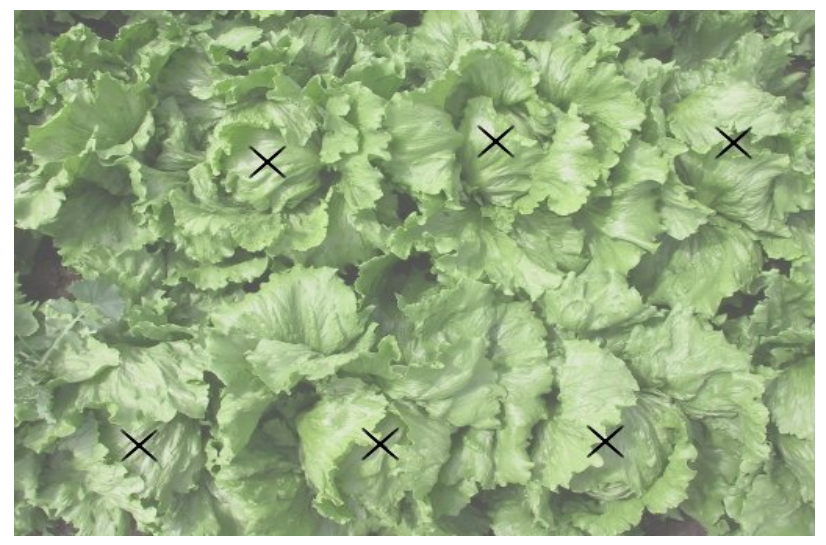

Fig. 2. Image of lettuce with lettuce locations marked with crosses

robust and sophisticated system will be investigated further.

A Laser Range Finder is used by [7] to localize spherical oranges on a tree. However their method differs in that range information is combined with attenuation information to determine colour properties to aid in recognition. Oranges show good contrast compared with the green to brown trees they grow on. They are near spherical and so are more easily recognized compared with lettuce or other similar vegetation.

Section II describes the general approach we have taken to recognize the lettuce. The collection and preprocessing of the lettuce range datasets is described in Section III. In Section IV we describe in detail the methods used to analyze the datasets, extract the significant features and construct the lettuce model to be used with an error function. The results of this method are presented in Section V. The advantages and limitations of this technique is discussed in Section VI.

\section{APPROACH}

A SICK Laser Range Finder was mounted above a lettuce field and was used to generate height plots of the lettuce. This provided information about the spatial distribution of the heights of the lettuce plants. The locations of the lettuce were recorded and then the appropriate regions of the height plots were extracted to act as the lettuce training example set. A similar number of regions was chosen at random to provide a training set of negative lettuce example regions that would be used to compare the discriminatory abilities of extracted features.

Due to the approximately circular nature of the lettuce, a radial distribution was found to provide the best and most consistent information about the relative height distribution. This method eliminates orientation from the distribution, vastly reducing the model complexity. Height distributions for discrete distances from the center of a region were calculated and histograms were used to represent this information. Histograms were also used to represent 3D shapes in [8]. They only used a single histogram of an entire $2 \mathrm{D}$ region, rather than also incorporating spatial information.

Various features and attributes were extracted from this set of distributions. Each attribute was tested for clustering within positive lettuce example scans and the for discrimination from negative lettuce examples. Four parameters were chosen and these formed the learned model of a lettuce.

Datasets with known lettuce locations were then used to verify the accuracy of the learned lettuce model. Regions were extracted sequentially from the dataset and the four attributes were calculated and used to describe that region in parameterized form. The error between the learned model vector and the region vector was found using a weighted Euclidean distance. Prior information of the row and inter row plant spacing was incorporated to reduce both false positives and false negatives. Regions of extremely low error were then assigned as lettuce locations.

\section{DATASETS}

\section{A. Experimental Setup}

In a future practical set up, a vehicle would be traversing the lettuce fields carrying out the gathering of laser range data. To emulate this situation a laser range finder mounted on a rail at a height ( $z$ coordinate) of $590 \mathrm{~mm}$ from the ground looking down at the top of the lettuce crop was traversed at increments of $10 \mathrm{~mm}$ (in $x$ direction) perpendicular to the scanning plane of the range finder. The laser range finder was set to an angular resolution of $0.5^{\circ}$ and the scanning range was set to $\pm 50^{\circ}$. This corresponds to a $y$ direction resolution of approximately $5 \mathrm{~mm}$. A total traversing distance of $2 \mathrm{~m}$ was covered for each plot. The number of plots traversed is 2 .

\section{B. Preprocessing}

As the laser range finder provided data in polar form, the data needed to be converted to cartesian form to allow for easy calculation of the radial distributions. This was done using standard trigonometric relations. The data was then interpolated to produce a $2 \mathrm{D}$ height grid with a resolution of $10 \mathrm{~mm}$ in the $x$ and $y$ directions, to aid in computation.

\section{ANALYSIS}

This section describes the techniques used to learn the attributes of lettuce examples and apply them to recognize lettuce in arbitrary datasets. 


\section{A. Generating Radial Distributions}

The lettuce examples were represented spatially as the height distribution against radial distance from the center of the lettuce. This was used as it provided a simpler and more descriptive representation than separate $\mathrm{X}$ and $\mathrm{Y}$ height distributions, due to the inherent quasi circular nature of the lettuce. Another advantage of the radial method is that it ignores the orientation of the lettuce to the sensor.

Each height point in the $300 \times 300 \mathrm{~mm}$ region was assigned a radial distance from the central point in the region. This distance was rounded to the nearest $10 \mathrm{~mm}$. The heights that fell in each radial segment (16 segments running from $0 \mathrm{~mm}-150 \mathrm{~mm}$ ) were grouped together to form the height distribution for that segment. Those heights that were further than $150 \mathrm{~mm}$ from the center were ignored.

\section{B. Normalizing to Remove Height Variation}

The heights in each region were locally normalized to lie between 0 and 1 so as to eliminate the overall variation in height of different lettuces. Some lettuces grew taller than others and thus had a higher mean height. The overall shape was similar and by normalizing the height, the variation between different lettuce were dramatically reduced.

\section{Choice of Attributes}

In order to attempt to learn the characteristic lettuce model it was necessary to determine a few key attributes that distinguish it. Ideally the representation would be as compact as possible both in the number of attributes and the representation of each attribute. This compactness is obviously advantageous from a computational point of view for real-time implementation. Also the principle of Ockham's Razor favours simpler models.

As a large number of the radial height distributions were approximately gaussian in nature, the mean and standard deviation of the distributions were chosen as obvious candidates. The positive and negative lettuce training sets were used to determine which of these attributes would be useful. This required that the lettuce examples cluster closely (low standard deviation) and that they discriminate between positive and negative examples (a significant difference between the means examples exists).

To quantify this usefulness, the Fisher ratio $F$ was proposed, as suggested in [1]. This formula quantifies the difference between a positive lettuce example distribution $l$ and a negative lettuce example distribution $\bar{l}$. To make the distinctions between useful and useless attributes clearer, the squared Fisher ratio $F^{2}$ was used instead.

$$
F^{2}=\left|\frac{\left(\bar{x}_{l}-\bar{x}_{\bar{l}}\right)^{2}}{\sigma_{l}^{2}+\sigma_{\bar{l}}^{2}}\right|
$$

The mean $\bar{x}$ and standard deviation $\sigma$ for each radial segment in each training example was found individually. The overall means $(\mu(\bar{x})$ and $\mu(\sigma))$ and standard deviations $(\sigma(\bar{x})$ and $\sigma(\sigma))$ for each radial segment were then calculated. These were used to find the squared Fisher ratios for each radial segment. The rule of thumb used was that values greater than 0.5 are useful, and values close to 0.0 are useless. Table I summarizes this information.

TABLE I

SUMMARY OF RADIAL DISTRIBUTIONS

\begin{tabular}{|l|c|c|c|c|}
\hline \hline 10 & 0.81 & 2.43 & 0.069 & 1.02 \\
20 & 0.79 & 1.97 & 0.068 & 2.63 \\
30 & 0.76 & 2.14 & 0.093 & 2.67 \\
40 & 0.71 & 1.31 & 0.113 & 2.31 \\
50 & 0.66 & 0.73 & 0.150 & 1.37 \\
60 & 0.60 & 0.22 & 0.189 & 0.59 \\
70 & 0.54 & 0.007 & 0.227 & 0.057 \\
80 & 0.51 & 0.006 & 0.245 & 0.005 \\
90 & 0.50 & 0.033 & 0.259 & 0.061 \\
\hline
\end{tabular}

Note that these values were calculated after normalization and the removal of outlier points as mentioned in the next section. The values for radial segment $0 \mathrm{~mm}$ are not shown as this distribution consists of a single point and so standard deviations are meaningless, and the sample size of 1 is far too small to be useful.

Table I shows that possible attributes are the means of radial segments from $0 \mathrm{~mm}$ to $50 \mathrm{~mm}$ and the standard deviations of radial segments from $0 \mathrm{~mm}$ to $60 \mathrm{~mm}$.

To minimize the number of attributes it was decided to combine neighboring radial segments into larger segments. This also has the benefit of increasing the number of points in each distribution and thus minimizing the effects of noisy measurements. The segments chosen to combine were $0 \mathrm{~mm}$ to $30 \mathrm{~mm}$ and $40 \mathrm{~mm}$ to $50 \mathrm{~mm}$ for both the mean and standard deviations, as the mean values were similar over each of these ranges. Therefore combining them will have minimal effect on their discriminatory value. This provides a compact representation for the lettuce model $l_{\mu}$, which consists of a vector containing only 4 parameters.

$$
l_{\mu}=\left[\mu\left(\bar{x}_{0-30}\right) \quad \mu\left(\bar{x}_{40-50}\right) \quad \mu\left(\sigma_{0-30}\right) \quad \mu\left(\sigma_{40-50}\right)\right]
$$

For later use as a scaling factor in the error calculations, the standard deviations $l_{\sigma}$ of these four parameters were also recorded along with their means. This effectively makes the representation into eight parameters.

$$
l_{\sigma}=\left[\begin{array}{llll}
\sigma\left(\bar{x}_{0-30}\right) & \sigma\left(\bar{x}_{40-50}\right) & \sigma\left(\sigma_{0-30}\right) & \sigma\left(\sigma_{40-50}\right)
\end{array}\right]
$$

\section{Removing Outlier Points}

Another preprocessing technique that was found to be very useful was to remove the outlier points from each region before calculating the region's distributions and descriptors. Heights that were further than 1.28 standard deviations away from the mean were eliminated. This value was chosen as it will eliminate the top and bottom $10 \%$ of points on a normal distribution. This is appropriate as the majority of the useful radial distributions can be reasonably approximated by a normal distribution.

This had the effect of dramatically improving the discriminatory value of the attributes. Some of the lettuce examples 
were skewed as they contained unusually high leaves, and this distorted the normalized distributions. On those lettuce examples that were not skewed and the examples of arbitrary regions, this technique had minimal effect on the distributions and descriptors as they contained few outliers.

Table II shows the effect of removing the top outliers and the bottom outliers using 1.28 standard deviations from the mean value as the threshold. The values shown are for the $0-30 \mathrm{~mm}$ mean radial distribution. Similar trends were seen in the other radial distributions. The Squared Fisher Ratio was again used to determine the relative change in discriminatory ability. This shows that by removing only the top $10 \%$ outliers, the greatest improvement was to be gained.

\section{TABLE II}

EFFECT OF REMOVING OUTLIERS FROM 0-30MM RADIAL DISTRIBUTION

\begin{tabular}{|l|c|c|}
\hline Outliers Removed & $F^{2}(\bar{x})$ & $F^{2}(\sigma)$ \\
\hline None & 1.62 & 1.08 \\
Top 10\% & 2.03 & 2.68 \\
Bottom 10\% & 1.50 & 0.045 \\
Top and Bottom 10\% & 1.77 & 0.91 \\
\hline
\end{tabular}

\section{E. Error Function}

To recognize arbitrary regions as lettuce, an error function was used. This function is based on the weighted vector distance between the arbitrary region parameter vector $r_{\mu}$ and the learned lettuce model vector $l_{\mu}$. The distance function used was the Euclidean Distance. The error of each attribute was scaled by the lettuce model standard deviations $l_{\sigma}$.

$$
E=\sqrt{\sum_{i} w_{i}\left(\frac{r_{i}-l_{i}}{\sigma_{i}}\right)^{2}}
$$

The error terms were weighted using a weight vector $w$. The values below were found to work well in practice. They are roughly based on the Squared Fisher Ratios.

$$
w=\left[\begin{array}{llll}
1 & 1 & 5 & 5
\end{array}\right]
$$

The standard deviation errors were weighted higher than the mean error terms as they were shown to have a much higher detection rate than the mean errors. However they also gave a large number of false positive detections. The mean error on the other hand, while having a lower detection ability, was a more reliable detector. Thus the weighted combination of the errors was used and showed better performance than either individual error plot.

The use of prior knowledge of the plant spacing, as described below, removed much of the need for the mean terms as it was able to successfully remove the majority of the false positives detected by the standard deviation error terms. However the mean terms were retained for now due to the risk of overfitting by removing them. A larger sample will be required to verify this.

Another improvement to the error function involved the standard deviation error terms. Intuitively low standard deviation is desirable, yet a segment with lower standard deviation than the lettuce model was unfairly punished. A dead zone was introduced to the standard deviation error terms so that the error component would only be non-zero if the sample standard deviation was higher than that of the trained model, as can be seen in Figure 3. This had the dramatic effect of solidifying the low error clusters associated with actual lettuce locations rather than having a ring of low error with higher error in the very center, as can be seen in Figure 4. Darker regions correspond to lower error and so more likely to be a lettuce.

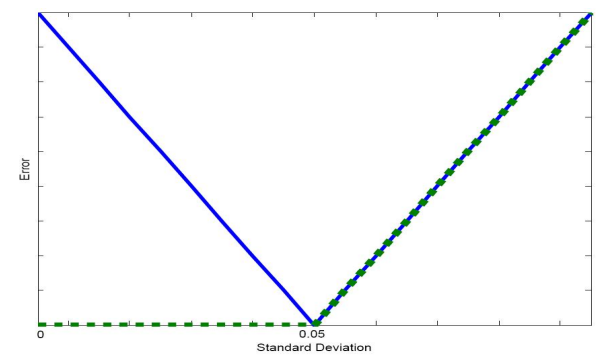

Fig. 3. Standard deviation $\sigma$ error deadzone. The solid blue line shows error as sample $\sigma$ varies. Lettuce model $\sigma$ in this case is at 0.05 and is the point of 0 error. The dashed green line shows the deadzone where the error is 0 when the sample $\sigma$ is less than the model $\sigma$.
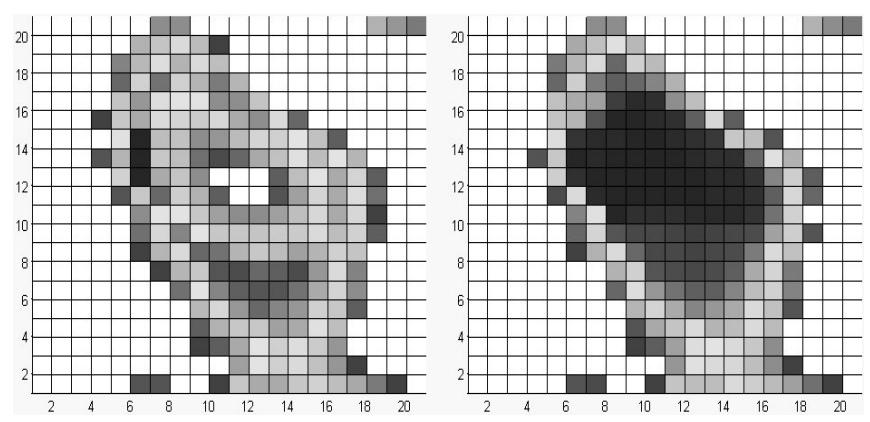

Fig. 4. The addition of dead zones to the standard deviation error terms solidified the low error clusters. The left plot is without the dead zones, while the right plot uses a dead zone. Darker red regions have lower error.

\section{F. Location of Lettuce in Error Plot}

Lettuces were located by assigning clusters of very low error to being lettuces. However the size, shape and error magnitude varied greatly amongst the lettuces and were also similar to some non lettuce regions as well. These areas of low error were used as a starting point and plant spacing information was used to distinguish between areas that were lettuce and those that were not. Figure 6 shows the lettuce scan before (left) and after (right) the error function has been applied. Dark clusters show likely lettuce locations.

\section{G. Planting Distance Information}

The lettuce were planted in rows at regular intervals. While they were seeded very accurately, as the lettuce grew, they grew away from the original planting location in a semi random direction. Variables such as the direction of sunlight may bias this but for simplicity it was assumed that this 

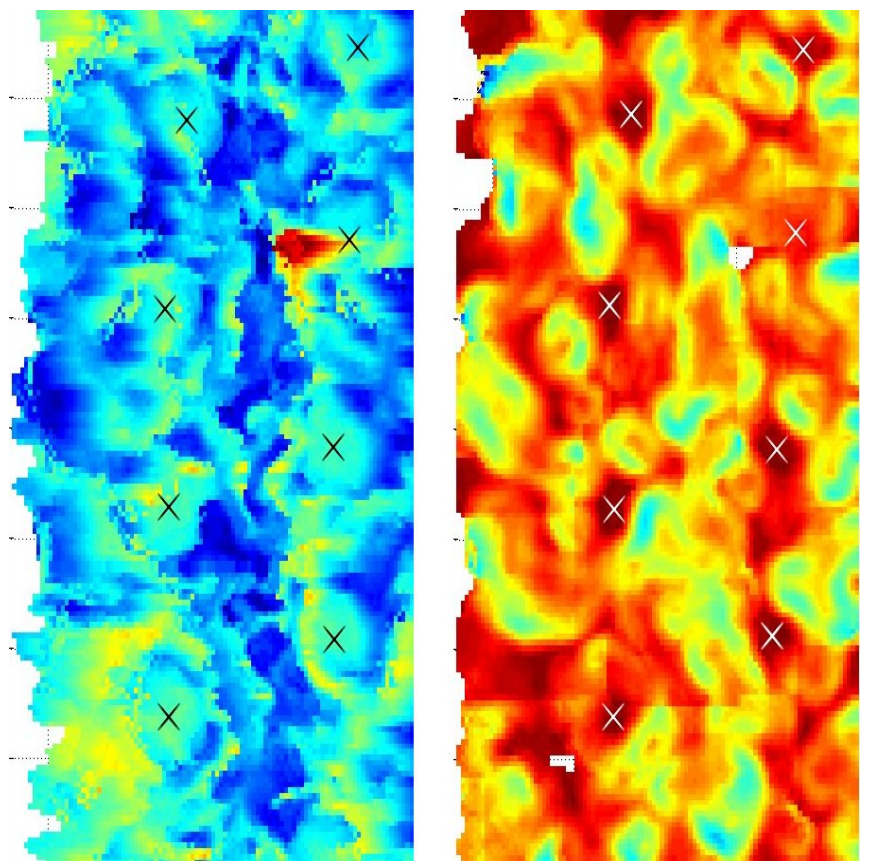

Fig. 5. The left plot shows the original laser height plot. The right plot shows the error function values with dark red being lowest error. The locations of lettuce are marked with crosses.

had a negligible effect. It is known as prior knowledge that the rows were planted $400 \mathrm{~mm}$ apart. This information was used to look for clusters of potential lettuce locations that lie within the same row and that are also approximately $400 \mathrm{~mm}$ away from similar bands of clusters.

The error plot was normalized and then inverted so that the most likely lettuce locations had a value of 1.0 , with 0.0 being least likely. A simple segmentation was performed with a threshold of 0.5 being used. A histogram was found by summing the plot along the $\mathrm{Y}$ direction. Assuming the lettuces in each row had approximately the same $\mathrm{X}$ position, peaks in this histogram should correspond to the position of rows of lettuce. This is similar to the method used for row detection by [9] where they summed grayscale image intensities to detect rows as the peaks.

Due to the false positives present in the error plot there was likely to be more peaks than actual rows. To determine which peaks corresponded to rows, the weight and spacing relative to other peaks were taken into account. The histogram peak detection algorithm used was based on ideas from [10] where they used a Multiresolution Peak Detection Algorithm.

These detected rows were then used to mask the error plot to remove many of the false positives that did not occur within a planted row. The mask used was trapezoidal in shape.

This process was then repeated within each row to locate the $y$ position of the lettuces within each row. The row was extracted, the threshold applied, and the region was summed in the $x$ direction. The peaks in the $y$ direction were found using the same peak detection method as for the rows. Those peaks of significant height and being consistently spaced were chosen as being the $y$ coordinate of a lettuce. For each of these $y$ coordinates, a smaller window of $y \pm 50 \mathrm{~mm}$ was extracted from the row and the single peak within the histogram of this region was chosen to be the $x$ coordinate of that lettuce.

\section{RESULTS}

The algorithm was run through two lettuce plot scans. Each scan consisted of 8 lettuces of marked locations, which were used as the training examples for the other plot. While this is not ideal for testing purposes, should these results show promise then a large dataset will be procured that will be used to validate the method and results. The results, while pending this further validation, are presented here to demonstrate the potential of this technique. This matter will be discussed further later.

\section{A. Lettuce Scan 1}

Table III shows that all eight lettuces were located with an average position error of $35 \mathrm{~mm}$. The first two columns are the actual lettuce positions, while the next two columns show the positions found by the algorithm. The ninth lettuce found at first appears to be a false positive, but upon inspection of the original scan, it is indeed a ninth lettuce. However as it was not initially recorded as such its exact position is unknown and so its exact error is also unknown.

TABLE III

LETTUCE SCAN 1 RESULTS

\begin{tabular}{|c|c|c|c|c|}
\hline $\mathrm{X}(\mathrm{mm})$ & $\mathrm{Y}(\mathrm{mm})$ & Alg X $(\mathrm{mm})$ & Alg Y $(\mathrm{mm})$ & Error $(\mathrm{mm})$ \\
\hline 400 & 270 & 390 & 280 & 14.1 \\
410 & 640 & 390 & 670 & 36.1 \\
400 & 1010 & 390 & 1040 & 31.6 \\
450 & 1360 & 420 & 1370 & 31.6 \\
810 & 430 & 770 & 430 & 40 \\
800 & 760 & 780 & 770 & 22.4 \\
840 & 1150 & 820 & 1110 & 44.7 \\
870 & 1490 & 810 & 1510 & 63.2 \\
- & - & 770 & 10 & - \\
\hline
\end{tabular}

\section{B. Lettuce Scan 2}

Table IV shows that seven out of the eight lettuces in the plot were successfully located with an average position error of $38 \mathrm{~mm}$. The eighth lettuce that was not located, did present a strong cluster within the original error plot, however it had grown substantially to one side of the row. This meant that when the rows were found and the mask was applied, the majority of this lettuce was removed from the row and so the algorithm failed to find it.

\section{Overall Results}

From the two plots used, 15 out of the 16 lettuces were located successfully with an average position error of $36.5 \mathrm{~mm}$. The average lettuce diameter was $170-200 \mathrm{~mm}$ so this error is reasonable. Excluding the ninth lettuce located in scan 1, there were no false positive detections. The spread of the position errors are shown in Figure 6. 
TABLE IV

LetTuCE SCAN 2 Results

\begin{tabular}{|c|c|c|c|c|}
\hline $\mathrm{X}(\mathrm{mm})$ & $\mathrm{Y}(\mathrm{mm})$ & Alg X $(\mathrm{mm})$ & Alg Y $(\mathrm{mm})$ & Error $(\mathrm{mm})$ \\
\hline 270 & 200 & 260 & 210 & 14.1 \\
280 & 540 & 220 & 530 & 60.8 \\
260 & 940 & 230 & 930 & 31.6 \\
280 & 1290 & 260 & 1240 & 53.9 \\
550 & 310 & - & - & - \\
650 & 660 & 640 & 660 & 10 \\
670 & 1060 & 650 & 990 & 72.8 \\
680 & 1360 & 680 & 1380 & 20 \\
\hline
\end{tabular}

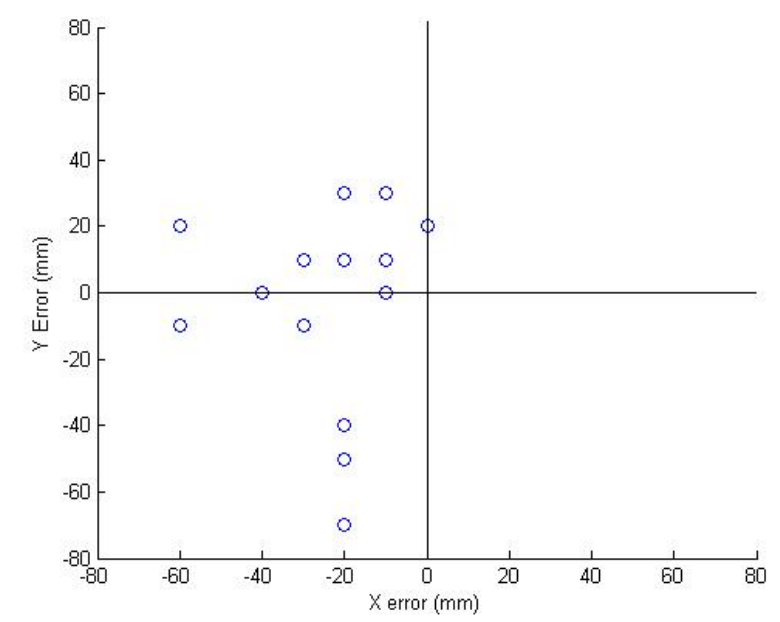

Fig. 6. Algorithm Location Error in $\mathrm{X}$ and $\mathrm{Y}$ directions

\section{DISCUSSION}

\section{A. Robustness of Algorithm}

Despite the successful recognition of the lettuce by this algorithm, it should be noted that the dataset used was small and so a test set of reasonable size could not be used. The dataset used consisted of two laser scans with 8 lettuce each. All 16 were used as training examples and test sets. However to mitigate this somewhat, two runs were performed with one scan being the training set and the other the set and vice versa. The algorithm still runs the risk of having overfit the training data and providing overly optimistic results. A larger dataset is currently being sought and will be used to verify the true accuracy and robustness of this algorithm.

The algorithm currently relies on all the lettuces being at approximately the same maturity level as each other and as the training examples. This will usually be the case for lettuces in the same field, however in its present form the algorithm will most likely need to be calibrated for different lettuce fields. This could be done by providing it with lettuce locations from that field to learn the lettuce model based on the particular characteristics of that field such as lettuce species, maturity level and planting distances.

The peak detection method is not optimal over the 2D error plot as it finds the peaks in each axis individually, so the peak found may not be the true peak. It is possible that more sophisticated methods such as finding the centroid of the error plot would improve the accuracy of this system.
The row mask used was also too restrictive. Examination of the error plots for those lettuce located with high location error revealed that while the lettuce were prominent in the original error plot, the mask had marginalised them as they were significantly offset from the row. A better method of masking the rows is needed to greatly improve the accuracy of these outlying cases.

\section{CONCLUSIONS}

The algorithm presented was shown to have promising performance when attempting to localize lettuce in the field. The use of spatial information from a laser range finder overcame the difficulties involved with using visual colour information due to the occlusion of lettuces by their leaves along with those of neighboring lettuces. The use of radial distributions to model a lettuce combined with prior information of row and interplant spacings proved successful in locating 15 out of 16 lettuces with a mean accuracy of $36.5 \mathrm{~mm}$.

It was noted that the generality and robustness of this technique are areas where further work is required. A larger dataset is being sought and will be used to validate the results. In order for this technique to be used in a real system, improvements to the algorithm, in several areas as previously noted, will be required to ensure its robustness and real time capabilities. However the original aim of proving the feasibility of this approach has been achieved and the use spatial information appears to be an area of promise in difficult localisation cases such as this.

\section{ACKNOWLEDGMENTS}

This work is supported in part by the ARC Centre of Excellence programme, funded by the Australian Research Council (ARC) and the New South Wales State Government.

\section{REFERENCES}

[1] A. Perez, F. Lopez, J. Benlloch, and S. Christensen, "Colour and shape analysis techniques for weed detection in cereal fields," Computers and Electronics in Agriculture, vol. 25, no. 3, pp. 197-212, 2000.

[2] B. Steward and L. Tian, "Real-time machine vision weed sensing," Urbana, vol. 51, p. 61801.

[3] W. Lee, D. Slaughter, and D. Giles, "Robotic Weed Control System for Tomatoes," Precision Agriculture, vol. 1, no. 1, pp. 95-113, 1999.

[4] B. Åstrand and A. Baerveldt, "An Agricultural Mobile Robot with Vision-Based Perception for Mechanical Weed Control," Autonomous Robots, vol. 13, no. 1, pp. 21-35, 2002.

[5] J. Blasco, J. Benlloch, M. Agusti, and E. Molto, "Machine vision for precise control of weeds," Proc. SPIE, pp. 336-343, 1998.

[6] A. Milella, G. Reina, M. Foglia, and A. Gentile, "Computer vision applications in agricultural robotics," 11th IEEE International Conference on Mechatronics and Machine Vision in Practice, Macau, 2004.

[7] A. Jimenez, A. Jain, R. Ceres, and J. Pons, "Automatic fruit recognition: a survey and new results using Range/Attenuation images," Pattern Recognition, vol. 32, no. 10, pp. 1719-1736, 1999.

[8] P. Lopes and E. Oliveira, "Solving partial occlusion with range images and 3D histograms," Industrial Electronics, Control, and Instrumentation, 1995., Proceedings of the 1995 IEEE IECON 21st International Conference on, vol. 2, 1995.

[9] H. Olsen, "Determination of row position in small-grain crops by analysis of video images," Computers and Electronics in Agriculture, vol. 12, no. 2, pp. 147-162, 1995.

[10] A. Guanshan Yu; Leen-Kiat Soh; Bond, "K-means clustering with multiresolution peak detection," Electro Information Technology, 2005 IEEE International Conference on, 2005. 